Aus der I. chirurgischen Abteilung des Rudolf-Virchow-Krankenhauses zu Berlin (Direktor: Prof. Dr. Hermes).

\title{
Zur Pathologie und Therapie der kongenitalen Nierendystopie ${ }^{1}$ ).
}

Von Dr. Aug. Lindemann, früherem Assistenzarzt der Abteilung.

Der bei beiden Geschlechtern auf Grund klinischer Untersuchungen nur selten erhobene Befund einer kongenitalen Dystopie einer oder beider Nieren, sowie die gelegentlich gemachte Beobachtung, daß krankhafte, durch die abnorme Lage des genannten Organes ausgelöste Prozesse, bezüglich ihres Ausgangspunktes und ihrer klinischen Bedeutung nicht genügend gewürdigt, zu größeren chirurgischen Eingriffen und damit unmittelbar zu ernsten Folgezuständen, ja selbst zum Tode geführt haben, veranlaßt mich, über einen in obengenannter Abteilung zur Operation und Heilung gelangten komplizierten Fall von Nierendystopie zu berichten und ihn in seinen Einzelheiten des näheren zu würdigen.

Es dürfte sich erübrigen, an dieser Stelle an Hand der aus der Literatur bekannten Fälle eine genaue Beschreibung der verschiedenen Arten von dystoper Niere zu geben und die Ursachen dieser Lageanomalie festzustellen, von der in der Kreuzbeinaushöhlung gelegenen „Kleinbeckenniere“ angefangen, bis zu den in und über Nabelhöhe fixierten Formen. Ich verweise in dieser Hinsicht auf die zusammenfassenden Abhandlungen von $\mathrm{K}$ e $\mathrm{h} \mathrm{r}$ e r, Scharogorodsky und Straeter, bei denen auch die übrige Literatur zusammengetragen ist, und möchte hier nur darauf hinweisen, daß die meisten Befunde von Nierendystopie zufällig gemacht wurden bei Gelegenheit von Autopsien, die wegen anderweitiger Erkrankungen bzw. Todesursachen ausgeführt wurden. Hier möge zunächst die Krankengeschichte unseres Patienten folgen:

I) Vortrag, gehalten im ärztlichen Verein zu Essen, Ruhr. 
Ein 23jähriger Spreeschiffer K., der bisher mit Ausnahme einer vor drei Jahren durchgemachten Infektion mit weichem Schanker und nachfolgender Leistendrüsenvereiterung immer gesund gewesen ist, erkrankt vier Tage vor der Aufnahme ins Krankenhaus. Am ersten Tage fühlt er sich morgens nicht recht wohl und erbricht im Laufe des Tages mehrmals. Unter Bettruhe bessert sich bis zum nächsten Tage das Befinden etwas; K. nimmt in dem Glauben, es handle sich bei ihm um einen einfachen Darmkatarrh, Rizinusöl. In den nächsten Stunden tritt von neuem Erbrechen ein, von mäßigen Schmerzen in der unteren Hälfte des Leibes begleitet. In der Nacht vor der Aufnahme ins Krankenhaus erfolgt noch mehrmals normale Stuhlentleerung, darnach stellt sich Harndrängen ein; will Patient demselben nachgeben, so kommt nur eine geringe Menge nicht sichtbar trüben Urins.

Bei der Aufnahme hat der Patient nur mäßige Schmerzen in der rechten Leibseite; befragt, wo dieselben beständen, zeigt er auf die Gegend des rechten Leistenkanals. Er ist ein junger, kräftig gebauter Mensch, Herz und Lungen zeigen keine krankhaften Veränderungen. Fieber ist nicht vorhanden, der Puls ist regelmäßig, kräftig, schlägt 7o mal in der Minute. Bei Betrachtung des Leibes (im Liegen) fällt auf, $\mathrm{da} B$ die Bauchwand oberhalb der Symphyse etwas mehr als anderswo hervorragt. Der Leib fühlt sich im ganzen weich an, nur entsprechend der Vorwölbung fühlt man oberhalb der Symphyse eine Geschwulst. Dieselbe reicht nach oben bis I Querfinger unterhalb des Nabels, grenzt sich hier und seitwärts in flacher Rundung ab. Neben derselben kann man tief mit dem palpierenden Finger eindringen, rechterseits zum Beispiel deutlich die Iliacalgefäße pulsieren fühlen. Die Ileocöcalgegend ist frei von abtastbaren Veränderungen. Per rectum fühlt man, ungefähr in der Mitte des kleinen Beckens gelegen, hoch oben eine flachkugelige Vorwölbung, die in ihrer Form einer gefüllten Blase entsprechen könnte. Sonst ist der Douglas frei, keine Infiltration der Wände fühlbar und keine Empfindlichkeit vorhanden. Die Geschwulst selbst ist bei Betastung vom Rektum aus in ganzer erreichbarer Ausdehnung, durch die Bauchwand hindurch nur am oberen rechten Ende, mäßig empfindlich. Es besteht über ihr deutliche Fluktuation.

Ein Katheterismus entleert Ioo $\mathrm{ccm}$ trüben Urins; zum Schluß folgen wenige Tropfen nach, die aber nicht trüber sind, wie die erstentleerte Partie. Eiweiß und Zucker sind nicht nachweisbar. Im Zentrifugat sind zahlreiche Leukocyten, keine Nierenelemente sichtbar. Nach dem Katheterismus ist die im Douglas fühlbar gewesene Geschwulst in gleicher Größe noch vorhanden. Die Zahl der Leukocyten im Blut beträgt am ersten Tage 5000, am zweiten Tage I0 000.

In den zwei ersten Tagen der Krankenhausbehandlung bleibt der Befund unverändert, Erbrechen tritt nicht auf, Stuhl erfolgt auf Einlauf, im Urin zeigt sich derselbe Befund wie bisher. Die Menge desselben beträgt am ersten Tage $850 \mathrm{ccm}$, am zweiten Tage $900 \mathrm{ccm}$ bei 
1020-1022 spez. Gewicht. Eine am dritten Tage vorgenommene Untersuchung ergiebt folgenden Befund: Nach Entleerung von $200 \mathrm{ccm}$ Urin durch Katheter aus der Blase sieht man oberhalb der Symphyse eine annähernd halbkugelige Geschwulst, welche nach oben bis 3 Querfinger unterhalb des Nabels reicht, die Bauchdecken vorwölbt und doppelte Mannsfaustgröße hat. Sie fühlt sich prall gespannt an und bietet deutliche Fluktuation. Die obere Umrandung derselben zeigt eine leicht dellenförmige Einsenkung. Nach unten setzt sich die Geschwulst in das kleine Becken hin fort, ist auch hier vom Rektum aus als halbkugelige Vorwölbung tastbar; auch hier bietet sie das Gefühl einer deutlichen Fluktuation. Die Geschwulst ist gegen die Unterlage nur sehr wenig beweglich, die Bauchdecken sind über ihr frei verschieblich. Der Klopfschall über derselben ist völlig leer. Die Palpation vom Rektum aus und durch die Bauchwand hindurch ist nur mäßig empfindlich, an der rechten oberen Kante dagegen wird ein mäßiger Schmerz geäußert. Das übrige Abdomen ist nicht aufgetrieben, überall weich und eindrückbar, nicht empfindlich; der Schall über demselben ist tympanitisch, die untere Leberdämpfung reicht bis zur Höhe des Rippenbogens, ein freier Erguß ist in der Bauchhöhle nicht nachzuweisen.

In der Annahme eines entzündlichen Tumors, der in Anbetracht des vorherrschenden Symptomenkomplexes mit großer Wahrscheinlichkeit mit einer der beiden Nieren in Zusammenhang zu bringen ist, wird (am dritten Krankheitstage) eine Laparotomie vorgenommen. Durch einen Medianschnitt in der Linea alba vom Nabel bis zur Symphyse werden die Bauchdecken durchtrennt, später der Schnitt zwecks freierer Übersicht noch nach oben um 2 Querfinger verlängert. Nach Eröffnung des Abdomens (freier Erguß ist nicht vorhanden) und Beiseiteschieben der vorliegenden, regelrecht aussehenden Dünndarmschlingen zeigt sich, der Wirbelsäule bzw. dem Kreuzbein aufliegend, eine von normalem Peritoneum überkleidete Geschwulst, über deren Herkunft vorderhand noch keine Sicherheit zu erlangen ist. Nach Abstopfen der Peritonealhöhle ringsum wird das hintere Peritonealblatt durch einen quer über die Geschwulst hinweggeführten Schnitt eröffnet; darunter kommt jetzt ein cystischer Tumor zum Vorschein, dessen Oberfläche den Eindruck erweckt, als handle es sich um Nierengewebe. Die in die Oberbauchgegend eingeführte Hand konstatiert mit Sicherheit das Vorhandensein einer normal großen linken Niere, während rechterseits eine solche nicht gefühlt werden kann. Da nun die Wandung des Tumors in seiner vorderen unteren, der Nierenkonvexität entsprechenden Hälfte sehr dünn und stellenweise fast durchscheinend ist, normal funktionierendes Nierengewebe also nur in sehr verringertem Maße vorhanden sein kann, außerdem drei auf der Oberfläche sichtbare frische, anämische Infarkte und zahlreiche bis schrotkugelgroße frische Hämorrhagien (siehe unten) auf eine ernste Schädigung des noch vorhandenen Nierenparenchyms hindeuten, so wird zur Exstir- 
pation der Geschwulst geschritten, zumal auch die in den letzten zwei Tagen entleerte Urinmenge $\left(\mathrm{I} 75^{\circ} \mathrm{ccm}\right)$ bei gleichbleibender Größe des Tumors auf ein genügendes Funktionieren der linken Niere hinweist. Die Ausschälung gelingt im allgemeinen leicht; nur nach dem kleinen Becken zu bestehen festere Adhäsionen. Beim stumpfen Loslösen derselben reißt die dünne Tumorwand ein, es entleert sich eine reichliche Menge getrübter, urinös riechender Flüssigkeit. Nach Austupfen derselben wird die Ausschälung vollendet, wobei nur einige fester an der Umgebung haftende Kapselteile zurückgelassen werden; man gelangt an den Nierenhilus, der nach hinten oben (dem Kreuzbein zu) und etwas nach rechts zu gelegen ist, und mehrere abgehende Gefäße, sowie einen Ureter erkennen läßt. Von den ersteren läßt sich eines (Arterie!) bis zur rechten Arteria iliaca communis verfolgen, der Uerter verliert sich abwärts im retroperitonealen Gewebe, ist im übrigen von normaler Dicke und Aussehen und verläuft, soweit sichtbar, ebenso wie die Gefäße ziemlich gradlinig. Durch isolierte Ligaturen werden zunächst die Gefäße versorgt, dann nach ihrer Durchtrennung noch eine Gesamtligatur um sie gelegt. Der Ureter wird einzeln unterbunden und durchtrennt. Nunmehr wird der Schlitz im hinteren Peritoneum durch eine Anzahl Nähte verkleinert, die übrig bleibende Offnung mit dem Peritoneum der vorderen Bauchwand vernäht. In den retroperitonealen Raum wird eine Schürzentamponade eingeführt, die Bauchwunde im übrigen durch Schichtnähte geschlossen.

Der Heilverlauf ist ein glatter. Höhere Temperaturen treten nicht auf. Sechs Tage nach der Operation kann der Schürzeninhalt, vier Tage darnach die Schürze selbst entfernt werden, die Wunde schließt dann unter weiterer lockerer Tamponade bei mäßiger Sekretion ziemlich rasch. 5 $\frac{1}{2}$ Wochen nach der Operation kann Patient mit leicht eingezogener fester Narbe, in deren. Mitte noch eine bohnengroße Granulationsfläche sichtbar ist, entlassen werden. - Die Urinmenge steigt am Tage nach der Operation auf IIoo ccm, in den nächsten Tagen bis $2000 \mathrm{ccm}$, das spezifische Gewicht geht auf normale Werte zurück. Pathologische Formelemente irgendwelcher Art lassen sich am Ende der Beobachtung nicht mehr nachweisen.

Soweit es unserem Patienten bekannt ist, hat er in früheren Jahren mit Ausnahme der schon erwähnten geschlechtlichen Infektion keine ernsten Krankheiten durchgemacht und sich bis zum vierten Tage vor der Krankenhausaufnahme ständig wohl gefühlt. Demgegenüber weist das Ergebnis, sowohl der klinischen Untersuchung, wie besonders der Operation, in nicht zu verkennender Weise aus Gründen, die im folgenden noch näher erörtert werden sollen, auf einen lange bestehenden, fehlerhaften Zustand seitens der rechten Niere hin, eine Art Entwicklungs- 
hemmung, die zeitlich noch den ersten Anfängen der intrauterinen Ausbildung zuzurechnen ist. Dieser beachtenswerte Umstand steht in Ubereinstimmung mit den von anderer Seite gemachten Beobachtungen, wonach eine Dystopie einer oder gar beider Nieren einmal als Nebenbefund, (und das schon öfter) bei Autopsien von Leuten verschiedensten Alters, oder aber, und das bisher erst in vereinzelten Fällen, durch klinische Untersuchungen sichergestellt wurde, während bis dahin nie diesbezügliche Beschwerden geäußert worden waren. Und es mußten erst Komplikationen eintreten, sei es seitens des an abnormer Stelle liegenden Organes selbst, sei es seitens der benachbarten (verdrängten) Organe, die zu klinisch erkennbaren Veränderungen führten und die fehlerhafte Lage aufdecken halfen.

Wenn nun an der Hand des vorliegenden Falles auf die Charakteristika der dystopen Niere hingewiesen werden soll, so käme als erstes Moment die Lage des Organes zum Körper in Frage. Bei unseren Patienten nahm letzteres bezüglich der Höhe eine Mittelstellung ein; es lag auf dem Promontorium, und zwar so, daß der Hilus schräg nach hinten oben zum Kreuzbein hinsah, die Konvexität nach der Bauchwand und unten hin gerichtet war; dabei stand das Organ im ganzen etwas schräg, so daß der obere Pol mehr nach links und oben, der untere mehr nach rechts und unten hin ging. Durch die bedeckende Peritonealschicht war das Organ in dieser Lage fest fixiert, so daß eine nennenswerte Verschieblichkeit gegen die Wirbelsäule, weder palpatorisch, noch in operatione nachzuweisen war. Diese Fixation war offenbar einmal dadurch bedingt, daß das Peritoneum nicht eine sackähnliche lockere Umhüllung bildete, mit einer dem Hilus entsprechenden (bruchsackhalsähnlichen) Verschmälerung, wie sie bisweilen auch in ausgebildeten Fällen von Ren mobilis erkennbar wird, sondern einen feststehenden straffen Überzug darstellte, der seitlich allseits nicht der Rundung der Nierenoberfläche entsprechend mitzog, sondern sich verflachend, den gewöhnlichen Verlauf der retroperitonealen Bekleidung nahm. Weiterhin trugen zur Befestigung des Organes die im Laufe der Zeit in den Zwischenräumen zwischen Peritoneum und Nierenoberfläche hier und $\mathrm{da}$, besonders an den abhängigen Partien entstandenen Schwarten bei, die eine erhebliche Festigkeit erlangt hatten, so daß ihre Durchtrennung stellenweise große 
Mühe gemacht hatte. Was die Entstehungsursache derselben anbelangt, so könnten sie sich ja im Gefolge interstitieller, in der Niere selbst sich abspielender Prozesse gebildet haben; dagegen spricht aber die gute Ablösbarkeit der Kapsel und das mikroskopische Bild der Nierensubstanz (siehe unten), die keinerlei Residuen eines interstitiellen Prozesses aufweist; vielmehr läßt sich eine genügende Erklärung für ihre Entstehung geben, wenn man eine immer wiederkehrende Reizung der Nierenoberfläche bzw. des sie bedeckenden, zunächst locker über sie hinwegziehenden Peritoneums annimmt, welche ihrerseits wieder bedingt ist durch den stets wechselnden, vom Füllungszustand der Eingeweide und Schwere des Organes selbst abhängigen ausgeübten Druck.

Es erscheint leicht verständlich, daß das Organ bezüglich seiner Gestalt nach Entwicklung und langem Verbleiben an fehlerhafter Stelle einen veränderten Anblick bot. Am ehesten glich es noch nach seiner Freilegung einem länglich ausgezogenen und von vorn nach hinten zusammengedrückten Gummiball, bei dem die breiteren Flächen dann der Nierenkonvexität und dem Hilus entsprechen. Bestimmend auf diese Änderung des Aussehens war offenbar der Druck, den die das Organ zwischen sich fassenden Gebilde auf dasselbe ausübten; auf der einen Seite das straffe Peritoneum, unterstützt durch die im Füllungszustande wechselnden Eingeweide, a uf der anderen Seite die unnachgiebige Wirbelsäule und das Kreuzbein. War derselbe bis zur Erkrankung des Patienten nur ein geringer, aber ständiger, so nahm er durch die in den ersten Tagen der Erkrankung einsetzende und bis zum Operationstage sich erheblich verstärkende Urinstauung im Nierenbecken, die. ihrerseits wieder eine erhebliche Vergrößerung des Organes zur Folge hatte, bedeutend zu.

Was die Größenmaße anbelangte, so betrug die Länge am gehärteten Präparate $16 \mathrm{~cm}$, die Breite $9 \mathrm{~cm}$, die Höhe $7 \mathrm{~cm}$, jedoch dürfte gerade die letztere Zahl etwas zu gering bewertet sein, da bei Ausschälung der unteren Hälfte des Organes bereits eine teilweise Entleerung des Nierenbeckeninhaltes stattgefunden hatte. Das Organ zeigte also eine erhebliche VergröBerung, die aber zweifellos nur auf die Verlegung des Nierenbeckenausganges zu beziehen war; ein wirkliches, bereits länger bestehendes Überschreiten der normalen Größenverhältnisse ging nicht mit Sicher- 
heit aus den genannten Zahlen hervor, ist auch, wie die bisher beobachteten Fälle von Nierendystopie erweisen, seltener. Gewöhnlich sind die dystopen Nieren, entsprechend ihrer verringerten Blutzufuhr (siehe unten), erheblich kleiner als normal, sei es nun, daß sie von Anfang an langsamer größer werden, oder daß sich eine immer mehr zunehmende Atrophie einstellt.

Die Nierenkapsel ließ sich im ganzen leicht ablösen, festere Adhäsionen mit der Rinde bestanden nur vereinzelt, dort, wo auch die Schwarten zu finden waren. Die Nierenoberfläche war im ganzen glatt, nur an einzelnen Stellen zeigten sich seichte Einsenkungen; tiefere Einkerbungen, wie sie sonst bei dystopen Nieren so häufig als Residuen der embryonalen Lappung gefunden wurden, waren nicht sichtbar. Ebensowenig waren, wie es bisweilen der Fall ist, Abdrücke von seiten der benachbarten Organe (Darm, Aorta, Wirbelsäule!) erkennbar.

Die Nierensubstanz war erheblich verdünnt. Ihre Dicke wechselte von I cm bis zu $2 \mathrm{~mm}$; besonders in den nach dem kleinen Becken zu gelegenen Teilen war sie sehr dünn und fast durchscheinend. An verschiedenen Stellen verteilt, zeigten sich auf den Schnittflächen drei größere, weißlich-graue, die Oberfläche etwas vorbuchtende Herde, die nach dem Nierenbecken zu sich verschmälerten und eine dunkelrote Randzone aufwiesen; der größte von ihnen zeigte eine Basis von $2 \frac{1}{2}: \mathrm{I} 1 / 2 \mathrm{~cm}$. Daneben waren noch zahlreiche kleine, dunkelrot aussehende Flecke auf der Oberfläche erkennbar, die nur $\mathrm{I}-2 \mathrm{~mm}$ in die Tiefe reichten. Die verschiedenen Schnittflächen boten im übrigen ein ziemlich gleichmäßiges, gelblich-graues Aussehen, nur vereinzelt waren gelbe Streifen erkennbar; die Grenzen von Rinde und Mark waren allseits verwaschen.

Der Nierenhilus hatte ebenso wie das ganze Organ seine Gestalt verändert; er war verbreitert und glatter geworden. Das Nierenbecken stellte sich nach dem Aufschneiden als eine große, die Gestalt des ganzen Organes in etwa nachahmende Höhlung dar; eine Mehrteilung, wie sie sich bisweilen bei Dystopie vorfindet, war nicht $\mathrm{zu}$ erkennen, ebenso wenig waren einzelne abgeteilte Buchten sichtbar; die Nierenkelche waren in der Höhlung stark abgeplattet, stellenweise kaum noch angedeutet. Der Inhalt des Nierenbeckens war eine trübe, dünne, eiterähnliche Flüssigkeit, 
deren Menge sich äußerer Gründe halber nicht mehr genau feststellen ließ. Irgendwelche Konkremente waren in ihr nicht vorhanden.

Aus dem Nierenbecken führte nach rechts und etwas schräg abwärts ein einzelner Gang, nach Form und Aussehen als Ureter anzusprechen, nicht erweitert und in seiner Wandung nicht verdickt. Er verlief (Operationssitus) im retroperitonealem Gewebe weiter nach schräg rechts und abwärts und verlor sich in diesem; seine Eintrittsstelle in die Blase wurde nicht sichtbar. Eine Schlängelung desselben war nicht erkennbar. Konkremente waren in ihm nicht zu fühlen.

Mehr nach der Mitte des Nierenhilus zu konnte man zwei weitere, mit engem Lumen versehene Gefäße erkennen, die sich bald nach dem Eintritt in den Hilus in Äste teilten und als Arterie und Vene anzusprechen waren. In ziemlich gradem Verlaufe, nicht geschlängelt, zogen sie nach rechts abwärts; das eine derselben, die Arterie, ließ sich bis zu seinem Eintritt in die Arteria iliaca dextra communis verfolgen. Ihr Inhalt bestand aus flüssigem Blut, auch in ihren größeren Ästen zeigten sich keine Gerinnsel. Beide Gefäße erschienen auffallend klein, im Gegensatz zu der sonstigen Dicke der Nierengefäße und der Größe des Organes, so daß der Verdacht bestand, daß außer ihnen noch weitere zuführende Gefäße vorhanden sein mußten. Es zeigten sich. denn auch am oberen Pole der Geschwulst noch zwei kleine Äste, deren weiterer Verlauf im Körper jedoch nicht festzustellen war. Am Präparat traten sie gleich nach ihrem Durchtritt durch die Kapsel in die Nierensubstanz ein.

Eine Nebenniere fand sich an dem herausgenommenen Organ nicht vor. Es ist das auch nicht überraschend, da die Nebennieren erst in späterer entwickelungsgeschichtlicher Zeit in eine rein örtliche Beziehung zu den vom Becken aufgestiegenen Nieren treten und bezüglich ihrer Anlage und Entwicklung nichts mit den einem anderen System zugehörigen Nieren.zu tun haben.

In einer großen Anzahl der bisherigen Veröffentlichungen über Nierendystopie wird auf das gleichzeitige Vorkommen von Anomalien des Urogenitalsystems verschiedenster Art und verschiedensten Grades hingewiesen, die sich besonders beim weiblichen 
Körper vorfänden und hier zumeist ihren Ausdruck fänden in Hypoplasie oder gar völligem Fehlen der betreffenden Organe, während beim männlichen Organismus nicht allzu selten sich neben anderen Mißbildungen eine Atresia ani verfände. Ich will auf diese interessanten Befunde hier nicht näher eingehen, zumal ich bei unserem Patienten nicht über Ähnliches berichten kann; es möge nur erwähnt sein, daß sie hinsichtlich ihrer Genese eine volle Erklärung finden in der innigen Zugehörigkeit der Nieren zum urogenitalen System in entwicklungsgeschichtlicher Hinsicht.

Dagegen möchte ich mit einigen Worten auf die bisweilen im dystopen Organ selbst beobachteten abnormen Bildungen eingehen. Hier kann es sich einmal um Mehrteilungen des Nierenbeckens infolge Fortbestehens fötaler Zustände handeln, so daß zwei oder gar drei voneinander bindegewebig abgetrennte Nierenbecken vorhanden sind, aus denen dann je ein Ureter nach abwärts zieht; entweder tritt dann eine Vereinigung vor der Einmündungsstelle in die Blase ein, odor (in selteneren Fällen) die Ureteren münden getrennt. Und weiter kann die Nierensubstanz selbst abnorme Bildungen aufweisen, von einfacheren cystenähnlichen $\mathrm{Ge}$ bilden angefangen, bis zu komplizierten Geschwulstformen, wo dann sowohl Gewebe vom Sarkomcharakter wie auch Drüsenschläuche zu sehen sind, in seltenen Fällen sich auch Knorpel und Knochen findet. - Bei unserem Patienten wies das Nierenbecken keine Besonderheiten in dieser Hinsicht auf; wohl die Nierensubstanz. Es zeigten sich in den zahlreich angefertigten Schnitten überall kleinste und größere, makroskopisch jedoch nicht mehr erkennbare Cysten; ihr Inhalt war zum größten Teile ausgefallen, das Epithel nur noch teilweise erhalten, der bindegewebige Grundstock jedoch deutlich erkennbar und viel reichlicher entwickelt, als es für gewöhnlich der Fall ist; außerdem zeigten sich in ihm zahlreiche elastische Fasern. Irgendwelche Prozesse narbiger Art, besonders nach den Spitzen der Nierenkelche hin, waren nicht zu erkennen, so daß es sich nicht um sekundäre, durch Abschnürung von Harnkanälchen entstandene Erweiterungen handeln konnte; außerdem wies gerade das Verhandensein der elastischen Fasern auf eine abnorme Anlage hin.

Was nun das Auftreten von pathologischen Prozessen in dem an abnormer Stelle liegenden Organ anbelangt, durch die bis- 
weilen erst die klinische Diagnose auf eine bestehende Nierendystopie hingelenkt wird, so hat man solche auf verschiedene Weise entstehen sehen; so besonders einmal durch Verlegung des Ureters oder der Gefäße, sei es durch Konkrementbildung oder durch mechanischen Druck seitens der das Organ umlagernden Gebilde; und ferner durch das Eindringen von Entzündungserregern in Nierenbecken oder Nierensubstanz, sei es durch Blase und Ureter, oder auf dem Wege der Blutbahn. Besteht allerdings der pathologische Proze $B$ schon länger, und ist er heftiger geworden, so lassen sich seine Anfänge bzw. Ursachen nicht mehr mit Sicherheit feststellen, selbst nicht am herausgenommenen Organ. So auch in unserem Falle; es zeigte sich zunächst eine infizierte Hydronephrose erheblichen Grades, die nicht durch einen Verschluß des Ureters durch Konkremente bedingt war; wohl aber wahrscheinlich durch eine Verlegung oder auch nur Verengerung desselben infolge Zusammendrückens seitens des Organes selbst. Weiter war das Nierenparenchym in allen angefertigten mikroskopischen Schnitten erheblich verändert. In fast sämtlichen Harnkanälchen lagen Zylinder, teils rein hyaliner Natur, teils mit Leukocyten bedeckt. Die Epithelien waren größtenteils ins Lumen abgestoßen, ihre Kerne nur noch stellenweise und schwach gefärbt. Das Zwischengewebe zeigte einmal, über das ganze Parenchym verteilt, einen vermehrten Reichtum an Leukocyten, dann waren letztere aber auch an umschriebener Stelle mehrfach in sehr zahlreicher Anzahl sichtbar, ohne da $B$ es jedoch schon zu einer Einschmelzung des Gewebes gekommen wäre. Die Gefäße, Arterien wie Venen, wiesen vermehrten Kernreichtum auf, die Arterien außerdem, entsprechend den schon erwähnten Herden, Thromben, die, ihrem Aussehen nach, schon einige Tage alt waren. Von den Herden selbst erwiesen sich die größeren als anämische Infarkte (völlige Farblosigkeit der Kerne, scholliger Zerfall der Epithelien) mit teils hämorrhagischer, teils Leukocyten-Randzone, die kleineren, oberflächlicheren als frische Infarcierungen. - Im ganzen also ein Bild schwerer parenchymatöser Entzündung, kompliziert durch multiple Infarcierung. Irgendwelche Bakterien konnten in den Schnittpräparaten nicht mehr nachgewiesen werden. (Eine bakteriologische Untersuchung der im Nierenbecken vorhanden gewesenen Flüssigkeit war nicht angestellt worden.) 
Die im vorhergesagten klargestellten Einzelbefunde, die sich (es sei hier nochmals kurz betont) im besonderen beziehen auf die Lage des Organes zum Körper, sein Verhalten zu der bedeckenden Peritonealschicht, auf die veränderte Form und das eigentümliche Verhalten von GefäBen und Ureter (zwei zu- und abführende relativ enge Gefäßpaare), von denen ein Arterienast aus der Arteria iliaca dextra communis gespeist wird, nichtgeschlängelter Verlauf der Gefäße und des Ureters) sowie auf gewisse Bildungen abnormer Art im Organ selbst, lassen mit Sicherheit erkennen, daB es sich um eine Dystopie der rechten Niere gehandelt hat, die erst lange Jahre nach ihrem Bestehen, gelegentlich einer eintretenden Entzündung, zur klinischen Erkenntnis kam.

Was die Differentialdiagnose gegenüber anderen klinisch sich ähnlich darstellenden Prozessen anbelangt, so ist ein sicherer Entscheid in dieser Hinsicht bisweilen sehr schwierig, wenn nicht gar unmöglich. Einmal kommen hier geschwulstartige Bildungen in Betracht, die vom Urogenitalsystem, vom Darm und seinen Anhängen oder von der Wirbelsäule oder den Beckenknochen ihren Ursprung nehmen (Sarkome, Carcinome, Cysten (Pankreas), Netztumoren). Oder es handelt sich um entzündliche Veränderungen, mögen sie nun akuten Charakter haben (Blinddarm, Blase) oder tuberkulösen Ursprungs sein (Senkungsabszesse). Als wichtige diagnostische Hilfsmittel kommen hier neben den gewohnten Untersuchungsmethoden in Betracht die Cysto- bzw. Ureteroskopie und die Röntgenoskopie, die, zumal wenn sie kombiniert ausgeführt werden, wohl mit Sicherheit die Diagnosenstellung ermöglichen. (Beide Methoden wurden äußerer Gründe halber hier nicht angewandt, die Diagnose blieb bis zur Stunde der Operation nur eine Wahrscheinlichkeitsdiagnose.)

Nun zur Therapie und damit auch zur Frage der Prognose noch einige Worte. Hier wäre zu entscheiden zwischen den Fällen, in denen das dystope Organ nur wenig oder gar keine Beschwerden macht und sekundäre Veränderungen nur geringer Art an ihm nachgewiesen werden können, und solchen.Fällen, in denen eine schwere Parenchymbenachteiligung des Organs außer Frage steht. Der alleinige Nachweis einer Dystopie einer der beiden Nieren dürfte wohl noch keine exakte Indikation zu einem Eingriffe irgend- 
welcher Art abgeben. Es sind nun mehrere Wege vorgeschlagen worden, die Dystopsie zu beseitigen bzw. Schädigungen vorzubeugen, die durch sie immerhin eintreten können. Einmal ist bereits öfter versucht worden, nach Lockerung des Organes aus seinem Bette und Verlagerung nach oben und der entsprechenden Seite hin eine Fixation vorzunehmen. Aber abgesehen davon, da $\beta$ ebensowenig, wie bei der Wanderniere durch einen derartigen Eingriff ein Dauererfolg garantiert werden kann, und bisweilen schon durch Knickung der Gefäße und des Ureters eine Verödung des Organes bzw. Stauung herbeigeführt worden ist, hindert an einer genügend weiten Verlagerung die Kürze der zum Organ ziehenden Gebilde. Außerdem paßt sich das in seiner Form meist schon erheblich veränderte Organ nur schwer der neuen Umgebung an und ruft neue Beschwerden hervor. Eine wirklich radikale Beseitigung der vorhandenen Beschwerden und der krankhaften Erscheinungen dürfte wohl nur die Nephrektomie bringen. Dieser Eingriff ist jedoch nur angezeigt für die Fälle, in denen es einmal, wie bei unserem Patienten, bereits zu Veränderungen schwerwiegender Art gekommen ist, also ein weiteres genügendes Funktionieren der (eventuell auch verlagerten) Niere nicht mehr gewährleistet werden kann, und dann auch eine zweite, genügend arbeitende Niere mit Sicherheit festgestellt worden ist (Ureteroskopie, Gefrierpunktsbestimmung). Sollte wegen Zeitmangels in letzterer Hinsicht eine klinische Untersuchung Sicheres nicht erbracht haben, so wäre zunächst die Freilegung der zweiten Niere oder ihre genaue Abtastung von der Operationswunde aus indiziert.

Wenn ich am Schlusse nochmals die Frage der Ätiologie der Erkrankung bei unserem Patienten berühren darf, so möchte ich bei Fehlen sonstiger Krankheitsursachen es für wahrscheinlich halten, daß es sich bei ihm, der den Schifferberuf ausübt, um eine von der Blase aufsteigende Infektion der rechten dystopen Niere gehandelt hat, die bei den in letzterer schon durch die abnorme Lage geänderten Strömungsverhältnissen rasch zu solch schweren Veränderungen führen konnte und auch geführt hat (Abknickung bzw. Verlegung des Ureters, Hydro-Pyonephrose, Nephritis, Infarcierung). Hätte es sich nur um einen mechanischen Verschluß des Nierenbeckenausganges bzw. des Ureters durch Druck oder 
608 Lindemann, Zur Pathologie und Therapie der kongenitalen Nierendystopie.

Stein und nachfolgende Infektion gehandelt, so wäre wohl das Nierenparenchym nicht schon in solch hochgradiger Veränderung begriffen gewesen.

\section{Literaturverzeichnis.}

I. K e h r e r, Beiträge zur Geburtshilfe und Gynäkologie. Festschrift für C h r o bek. Wien 1903 .

2. Scharogorodsky, M., Zur Würdigung der Nierendystopie in anatomischer und klinischer Beziehung. Inaug.-Diss. Berlin 1905.

3. Sträter, M., Beiträge zur Pathologie und. Therapie der kongenitalen Nierendystopie. Deutsche Zeitschr. f. Chirurgie 1906.

4. $S c h m a$ u $B$ und $K a$ u fmann, Lehrbücher der pathologischen Anatomie. 